\title{
DOCKING STUDIES FOR VARIOUS ANTIBACTERIAL BENZILATE DERIVATIVES
}

\author{
SUDHA R ${ }^{1 *}$, BRINDHA DEVI P², CHARLES C KANAKAM ${ }^{3}$, NITHYA G ${ }^{1}$
}

${ }^{1}$ Department of Chemistry, School of Basic Sciences, Vels University, Chennai, Tamil Nadu, India. ${ }^{2}$ Department of Bioengineering, School of Engineering, Vels University, Chennai, Tamil Nadu, India. ${ }^{3}$ Depatment of Chemistry, Formerly Presidency College, University of Madras, Tamil Nadu, India. Email: rajendran.sudha7@gmail.com

Received: 21 December 2016, Revised and Accepted: 12 January 2017

ABSTRACT

Objectives: In this study, we have focused on discovering the leads for the enzyme targets of infectious disease tuberculosis. We employed computeraided drug design docking tool, to discover new leads for Mycobacterium tuberculosis (MTB).

Methods: Five compounds were synthesized and they are made to dock into the active site of the enzyme; retrieved from protein data bank.

Results: The docking studies and structure-activity relationship reveals that the compound 2'-chloro-4-methoxy-3nitro benzilic acid after three different docking strategies reveals that the score was found to be higher compared with others $(-5.568 \mathrm{kcal} / \mathrm{mol})$.

Conclusion: On the closer analysis of this molecule, the molecule showed stacking interaction and the compound has also found to be surrounded by non-polar amino acids, which makes this molecule potent toward antibacterial drug discovery.

Keywords: Antibacterials, Docking, Absorption, Distribution, Metabolism and excretion study, Resistance.

(C) 2017 The Authors. Published by Innovare Academic Sciences Pvt Ltd. This is an open access article under the CC BY license (http://creativecommons. org/licenses/by/4. 0/) DOI: http://dx.doi.org/10.22159/ajpcr.2017.v10i4.16713

\section{INTRODUCTION}

As there is an increased number of drug-resistant bacterial cases worldwide, there is an urgent need for novel therapeutic interventions including innovative antibacterial and antimycobacterial drugs [1,2] with no cross-resistance to available drugs in the market. The most pathogenic bacteria Mycobacterium tuberculosis (MTB), the causative agent for tuberculosis in humans leads to a bacterial killer worldwide [3]. The bacteria have led to the emergence of multi-drug resistant and extensively drug resistant strains of bacteria. The treatment is based on the combination of two or more antibiotics, and the side effects are many. To limit the medications and side effects the preliminary studies have been done for the small molecule inhibitors which have been shown good antibacterial activity toward many pathogenic bacteria.

Recently, benzilic acid derivatives were synthesized as potent antibacterial agents with good activity range. They inhibit the pathogenic bacteria's which includes Staphylococcus aureus, Klebsiella pneumoniae, Escherichia coli, and Staphylococcus epidermitis. Virtual screening, a computational method where the compounds could be assessed for their potential to bind specific sites on target molecules such as proteins, was employed in the study [4]. Furthermore, the pharmacokinetic properties were also predicted for future perspective of the small molecule compounds.

\section{METHODS}

\section{Computational details}

The computational details for this study were conducted in an Intel Core I5 capacity processor with memory of $4 \mathrm{~GB}$ random access memory running with the windows 7 operating system. The virtual screening options for high throughput virtual screening (HTVS), standard precision (SP) and glide extra precision (XP) docking were all checked to be executed. The module glide XP of Schrodinger 9.3 (glide, version 5.7, Schrodinger, LLC, and New York, 2015) was utilized to perform docking studies. Suitable bonding and the charges were added to the hetero atoms and the corresponding hydrogen atoms were added to all the atoms [5].

\section{Protein preparation}

The protein file was prepared on protein preparation wizard and the energy minimization was performed. About 500 cycles of steepest descent and 5000 cycles of conjugate gradient methods with optimized potential for liquid simulations (OPLS) 2005 force field using Schrodinger suite version 9.3 were employed. Grid-A rectangular box surrounding the active site of the protein was located using receptor grid generation panel. The "Write XP descriptor information" option was selected and "compute root-mean-square deviation (RMSD)" option was enabled and rest of the parameters was kept as default. The XP glide scoring function was used to order the best-ranked compounds and the important interactions like $\pi$-cation and $\pi-\pi$ stacking were analyzed using XP visualizer in glide module. The input RMSD of the crystal ligand was also ascertained [6].

\section{Preparation of ligands}

The synthesized molecules were processed through the Lipinski filters to enable the drug property. Ligand preparation was performed for the synthesized molecules using LigPrep module available in the software (LigPrep v2.2, Schrodinger LLC, NY) and Epik (Epik v1.6, Schrodinger, LLC, NY) to expand protonation and tautomeric states at $7.0 \pm 2.0 \mathrm{pH}$ units. Conformational sampling was also performed for all database molecules using the Confgen search algorithm. Confgen with OPLS 2005 force field was applied for the generation of conformers with duplicate poses eliminate if the RMSD was $<2.0 \AA$. A distance-dependent dielectric constant of four and maximum relative energy difference of $10 \mathrm{kcal} / \mathrm{mol}$ were applied [6].

\section{Molecular docking}

Docking studies for the synthesized compounds were performed using glide module of Schrodinger, LLC, 2015. Primarily, using glide module $[7,8]$ (grid based ligand docking with energetics), we examined for important interactions based on the reference ligand and the protein of interest in the flexible mode docking. The glide module with three modes of docking, HTVS, SP, and XP mode was employed sequentially. The XP mode was used for exhaustive sampling and advanced scoring, resulting in even higher enrichment. 
Finally, the shortlisted hit molecules were selected based on the visual inspection of amino acid interaction, docking score, and the active site cavity [9].

\section{ADME prediction}

All the synthesized compounds for our study were selected, and the molecules were subjected to ADME predicted analysis using QikProp module of Schrodinger. The important properties such as octanolwater coefficient $(\log \mathrm{P})$, human oral absorption, Lipinski's rule of five, blood-brain barrier (BBB) coefficient, HERG property, and CaCO-2 permeability property were predicted for the synthesized compounds, and also the predicted results were checked for any violations to determine the nature of the compounds.

\section{RESULTS AND DISCUSSIONS}

Synthesized compounds have taken for docking studies to establish the structure-activity relationship using crystal structure of MTB co-crystallized with inhibitor thiazole benzamide (protein data bank ID:4WYC). Analysis of crystal structure of 4WYC revealed with hydrogen bonding interactions with nonpolar interaction like Trp398. The inhibitor is well associated with hydrophobic amino acids met61, Trp398, Trp64, Tyr407, and phe402 [10]. To validate the active site pocket the reference ligand was redocked and the docking score was found to be $-6.032 \mathrm{kcal} / \mathrm{mol}$. Redocking results showed that the compound exhibited similar interactions as that of crystal structure and showed an RMSD of $1.02 \AA$. Further, the compounds synthesized were screened based on three different docking strategies [11]. The ligand interaction with protein was depicted in Fig. 1. The docking score and the ligand interactions for the compounds were tabulated in Table 1.

The compound 2'-chloro-4-methoxy-3-nitro benzilic acid was found to inhibit the pathogenic bacteria's $S$. aureus, K. pneumoniae and E. coli at a distance of $10 \mathrm{~mm}$ using disc diffusion method, when compared to other compounds. The compound after three different docking strategies reveals that the score was found to be $-5.568 \mathrm{kcal} / \mathrm{mol}$. On the closer analysis of this molecule, the molecule showed similar stacking interaction like the reference molecule; the compound has also found to be surrounded by nonpolar amino acids, which makes this molecule potent toward antibacterial drug discovery. The binding analysis and ligand interaction diagram for the compound 2'-chloro-4methoxy-3-nitrobenzilic acid was depicted in Fig. 2.

Based on our docking studies, it has confirmed that the structure changes in the compounds series were found to be well correlated with in vitro antibacterial results. The compound 4, 4'-dibromo benzilic acid possessed high docking score of $-5.228 \mathrm{kcal} / \mathrm{mol}$ with the stacking interaction with amino acid Trp64. The activity of this compound also found to be well correlated with the reference ligand. The close analysis of this compound revealed that the compound is well packed with nonpolar interactions which make this compound more active against the pathogenic bacteria. The binding analysis and ligand interaction diagram for the most active compound 4, 4'-dibromo benzylic acid are shown in Fig. 3. The docking score and its ligand interaction for the synthesized compounds are tabulated in Table 1.

The compound 2, 2'-dichloro benzilic acid was found to inhibit the bacteria at a distance of $8 \mathrm{~mm}$. This is quite lesser than the other molecules. This makes this molecule more effective binding, and the docking score was found to be $-5.121 \mathrm{kcal} / \mathrm{mol}$. The binding analysis of this compound reveals that the compound well fitted into the active site pocket and the group phenyl chloride was found to interact with nonpolar amino acids Tyr25 and Trp64 which reveals that there are two stacking interactions making this compound more stable for further processing as better drug compound [12]. The binding analysis and ligand interaction for the compound 2, 2'-dichloro benzilic acid was depicted in Fig. 4.

The compound benzilic acid was found to inhibit the bacteria $K$. pneumoniae at a distance of $12 \mathrm{~mm}$. This compound after in silico screening analysis was found to possess good docking score $-5.069 \mathrm{kcal} / \mathrm{mol}$. On the closer analysis of this compound reveals that the molecule has well fitted into the active site pocket of the protein; also their ligand interaction shows that the molecule was surrounded by nonpolar amino acid and it is found to be interact with an amino acid Trp64 which is an important interaction of original ligand. The binding

Table 1: Docking score and ligand interaction results for the synthesized compounds

\begin{tabular}{llll}
\hline S. No. & Compound name & $\begin{array}{l}\text { Docking } \\
\text { score } \\
\text { kcal/mol }\end{array}$ & $\begin{array}{l}\text { Ligand } \\
\text { interaction }\end{array}$ \\
\hline 1 & $\begin{array}{l}\text { 2'-chloro-4-methoxy-3-nitro } \\
\text { benzilc acid }\end{array}$ & -5.568 & Trp64 \\
& 4, 4'-dibromo benzilic acid & -5.225 & Tyr157 \\
3 & 2, 2'-dichlorobenzilic acid & -5.121 & Trp64, Trp25 \\
4 & Benzilic acid & -5.069 & Trp64 \\
5 & Methyl benzilate & -3.140 & Trp64 \\
\hline
\end{tabular}

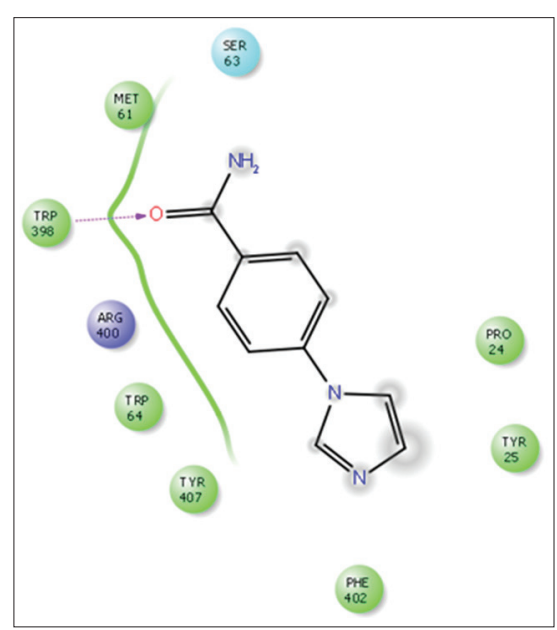

Fig. 1: Reference ligand interaction with protein

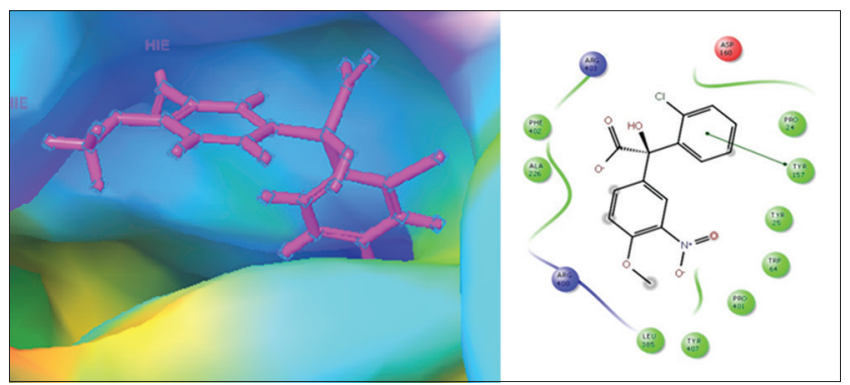

Fig. 2: Binding analysis and ligand interaction diagram for 2'-chloro-4-methoxy-3-nitro benzilic acid

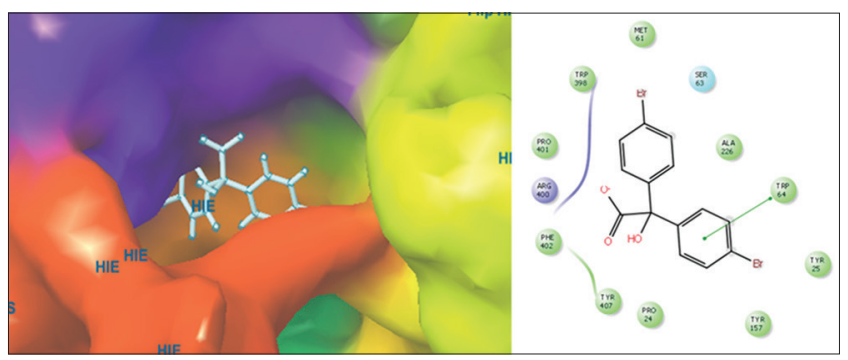

Fig. 3: Binding analysis and ligand interaction diagram for the most active compound 4, 4'-dibromo benzilic acid 
Table 2: ADME prediction for the synthesized compounds

\begin{tabular}{|c|c|c|c|c|c|}
\hline \multirow[t]{2}{*}{ Compound name } & \multirow{2}{*}{$\begin{array}{l}\text { QPlog } \\
\text { Po/wa }\end{array}$} & \multirow{2}{*}{$\begin{array}{c}\text { QPlog } \\
\text { HERG }^{\mathbf{b}}\end{array}$} & \multirow{2}{*}{$\begin{array}{l}\mathrm{QPP} \\
\mathrm{CaCO}^{\mathrm{c}}\end{array}$} & \multirow{2}{*}{$\begin{array}{l}\text { QPlog } \\
\text { BB }^{\text {d }}\end{array}$} & \multirow[t]{2}{*}{ Percent human oral absorption ${ }^{\mathrm{e}}$} \\
\hline & & & & & \\
\hline Methyl benzilate & 3.078 & -4.996 & 1558.995 & -0.403 & 100 \\
\hline 2'-chloro-4-methoxy-3-nitro benzilic acid & 3.078 & -4.996 & 1558.995 & -0.403 & 100 \\
\hline 4, 4'-dibromo benzilic acid & 4.199 & -3.195 & 222.95 & -0.303 & 93.562 \\
\hline Benzilic acid & 2.98 & -3.193 & 222.314 & -0.619 & 86.397 \\
\hline
\end{tabular}

aPredicted octanol/water partition coefficient logP (acceptable range: -2.0 to 6.5); ${ }^{\text {PPredicted }} \mathrm{IC}_{50}$ value for blockage of HERG K+ channels (below -5 ); ${ }^{\text {cPredicted }}$

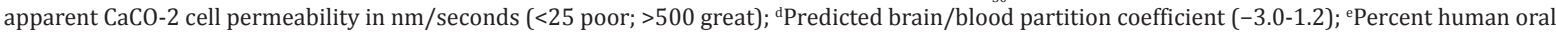
absorption ( $<25 \%$ is poor and $>80 \%$ is high); ${ }^{\text {fRule of } 5 \text { violation ( } m o l \_M W ~}<500$, QPlogPo/w $<5$, donorHB $\leq 5$, accptHB $\leq 10$ )

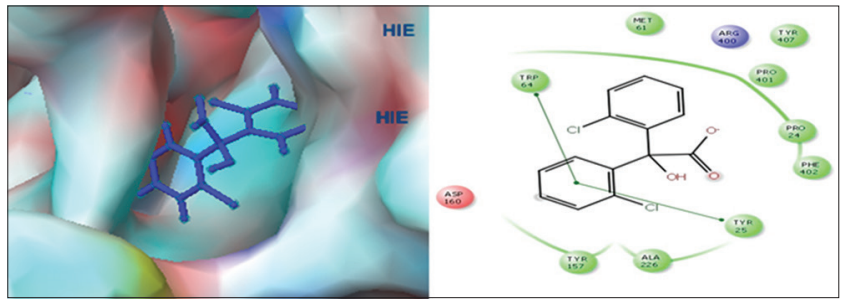

Fig. 4: Binding analysis and ligand interaction diagram for the most active compound 2, 2'-dichloro benzilic acid

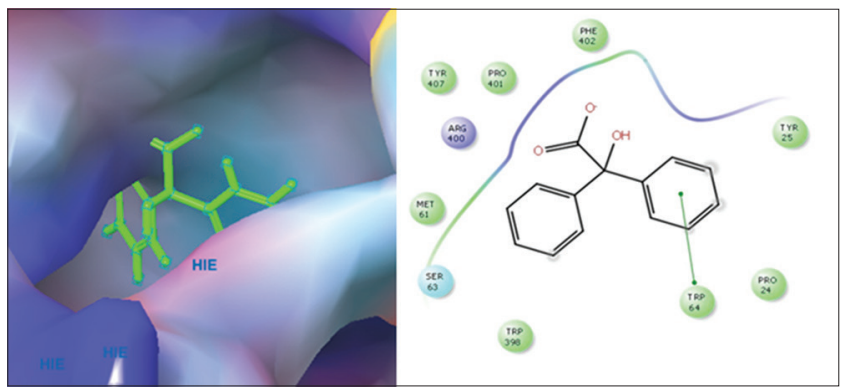

Fig. 5: Binding analysis and ligand interaction diagram for the most active compound benzilic acid

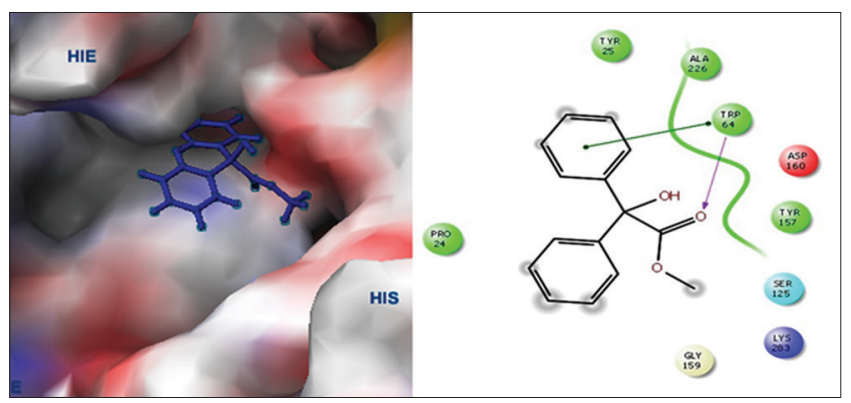

Fig. 6: Binding analysis and ligand interaction for the compound methyl benzilate

analysis and ligand interaction diagram for the compound methyl benzilate were depicted in Fig. 5 .

The compound methyl benzilate does not show any antibacterial activity with any of the pathogenic bacteria's. After molecular docking studies, the compound possesses less docking score $-3.140 \mathrm{kcal} / \mathrm{mol}$. The closer analysis of this reveals that the compound does not well fit into the active site of the enzyme. Furthermore, the benzyl moiety has facing outward which might have makes this compound less active when compared with other compounds virtually and biologically. The binding and ligand interaction for the compound methyl benzilate was shown in the Fig. 6.

\section{ADME prediction}

To further account for the potential of the compounds to act as efficient drug candidates, their absorption, distribution, metabolism, and excretion (ADME) properties were also calculated in silico using Qikprop. The obtained values for molecular logP, HERG property, CaCO accessibility, BBB, and human oral absorption; it is also used to assess violation of Lipinski's rule of five if any. All the compounds were shown to correlate well with the human oral absorption. BBB separates the human brain from the direct contact of circulatory system, thus protecting the brain for unwanted solute particles. Both the predicted compounds were shown to be BBB negative ensuring their administration safe for the brain. The ADME predictions for the synthesized compounds were tabulated in Table 2.

\section{CONCLUSIONS}

We utilized the medicinal chemistry tools of structure-based drug design strategy. Docking studies were performed to identify new scaffold molecules. This strategy revealed hitherto unknown binding pockets and inhibitor binding modes distinct from the earlier reported inhibitors and will be exploited successfully in further antimycobacterial drug development process. The most active compound 2'-chloro-4methoxy-3-nitro benzilic acid was found to be most active in both in silico and in vitro antibacterial analysis. Further, these compounds will be carried out for their antimycobacterial property as these are small molecule leads could easily cross the cell barrier systems in mycobacteria.

\section{ACKNOWLEDGMENT}

Authors thank Dr. Chandrasekar, Director, Department of Mechanical Engineering, Vels University for providing us the computer system with all the configuration which we needed for our study.

\section{REFERENCES}

1. Chandirana PR, Premnathb D, Kumara SV. Design, synthesis, molecular docking and antibacterial evaluation of novel n-(6, 11-dioxodihydro-5h-benzo [b] carbazol-2yl) benzamide derivatives as potent antibacterial agents. Int J Pharm Pharm Sci 2014;6(6):244-9.

2. Kumara KS, Krishnamurthy GA, Kumarn AS. Synthesis, characterization, in vitro antimicrobial, anthelmintic and docking studies of new 2-[(e)-\{[4-(1h-1, 2, 4-triazol-1 ylmethyl) phenyl] imino methyl] phenol, and their complexes with 3D metal ions. Int J Pharm Pharm Sci 2016;8(9):134-9.

3. Koul A, Arnoult E, Lounis N, Guillemont J, Andries K. The challenge of new drug discovery for tuberculosis. Nature 2011;469(7331):483-90.

4. Hajduk PJ, Greer J. A decade of fragment-based drug design: Strategic advances and lessons learned. Nat Rev Drug Discov 2007;6(3):211-9.

5. LLC. Maestro, Version 9.3. New York, NY: Schrödinger, LLC; 2015.

6. Saxena S, Devi PB, Soni V, Yogeeswari P, Sriram D. Identification of novel inhibitors against Mycobacterium tuberculosis L-alanine dehydrogenase (MTB-AlaDH) through structure-based virtual screening. J Mol Graph Model 2014;47:37-43.

7. Friesner RA, Banks JL, Murphy RB, Halgren TA, Klicic JJ, Mainz DT, et al. Glide: A new approach for rapid, accurate docking and scoring 1. Method and assessment of docking accuracy. J Med Chem 
2004;47(7):1739-49.

8. Kawatkar S, Wang H, Czrminski R, Joseph-Mclarthy D. Virtual fragment screening: An exploration of various docking and scoring protocols for fragments using Glide: J Comput Aided Mol Des 2009;23(8):527-39.

9. Alverez J, Shoichet B, editors. Virtual Screening in Drug Discovery. Boca Raton, Florida: Taylor Francis; 2005.

10. Dai R, Geders TW, Liu F, Park SW, Schnappinger D, Aldrich CC, et al.
Fragment-based exploration of binding site flexibility in Mycobacterium tuberculosis BioA. J Med Chem 2015;58(13):5208-17.

11. Sudha R, Kanakam CC, Nithya G. Synthesis, characterization and antimicrobial activity of substituted benzilic acids. Chem Tech 2015;8:383-7.

12. Jennings A, Tennant M. Discovery strategies in a BioPharmaceutical startup: Maximising your chances of success using computational filters. Curr Pharm Des 2005;11:335-44. 